\title{
Automated Image Segmentation and Analysis of Rock Piles in an Open-Pit Mine
}

\author{
Matthew J Thurley \\ Computer Science, Electrical and Space Engineering \\ Luleå University of Technology \\ Luleå, SWEDEN 97187 \\ Contact: http://www.ltu.se/staff/m/mjt, matthew.thurley@ltu.se
}

\begin{abstract}
Measurement and image analysis of 3D surface profile data of blasted rock piles in an open-pit mine are presented. A proof-of-concept/demonstration project into determining the size distribution of the visible rocks on the pile was performed. The results demonstrate the capacity to collect high resolution 3D surface profile data using a high-end two-axis scanning laser range-finder. Furthermore, automated image analysis was applied to this data to identify and size the rocks on the pile. Areas of very fine particles, too small to individually detect, are able to be detected and classified as areas-of-fines. Detection of these areasof-fines is extremely important as the amount of fine material is a key factor in evaluating blasting outcomes. The algorithms to perform this segmentation and classification analysis are outlined and results are shown in the form of images and sizing graphs.
\end{abstract}

\section{INTRODUCTION}

In the mining and aggregate industries a great many processes affect, and are effected by rock fragment (or particle) size, including blasting, comminution, and agglomeration processes. The particle size, or particle size distribution is a key factor influencing production rates and machine performance, in particular during the grinding and crushing of rock, and during excavation of rock piles. The comminution process defines the whole process chain of rock breakage and grinding to produce small particles from a large rock mass or ore body. This is a predominant process in mining where the aim is to convert the rock mass into particles small enough to liberate out the valuable minerals.

The process of mining is the phase of operation from blasting through excavating and hauling to delivery to a crusher or grinding mill. Once the material reaches the crusher or grinding mill the process is typically referred to as mineral processing. It is useful to make this distinction as the two phases are often separately managed and have different goals and demands. Mining traditionally focuses on delivering fragmented rock to the mineral processing plant at high rates to ensure downstream machinery is not waiting for material, but without a significant need for small particle sizes. Mineral processing seeks to have high throughput but can only achieve this by grinding the mineral laden rock down to small sizes.

Since the 1960's, papers in the field of blasting, fragmentation and mine optimisation have called for increased expenditure on drilling and blasting to produce smaller and more highly fractured fragments in order to reduce the downstream costs [1]. According to Grant et. al. [2] "once a hard-rock ore-body is defined the activity which has the most substantial impact in determining the efficiency of a mining operation is the blasting".

For the mine manager there are competing demands such as how to achieve high production rates, keep costs low, produce finer and more fractured rocks for the mineral processing plant, and to prevent excess damage to the mine which for example might weaken the open-pit and make it more susceptible to bench-failure or collapse.

It order to balance these demands and optimize mining it is necessary to test and measure, but whilst blasting is an advanced engineering and research discipline in its own right, the accepted technology for quantitatively measuring particle size still relies on the ancient technology of sieving or screening. Sieving is a process where rocks are passed through a series of progressively smaller (typically) square mesh screens whereupon the rocks are weighed and classified into size classes based on which screen they did not pass through. However, screening is typically impractical as a routine assessment tool due to slow feedback, inconsistent measurement, time consuming interruption, and is certainly impractical for full scale blasted rock piles in the mine.

As a result there is an opportunity for automated machine vision systems for measurement of the particle size distribution that can provide a quantitative, robust measurement to facilitate large-scale, comparative studies, blasting optimizations and automatic control optimizations in the mineral processing plant.

Particle size measurement using machine vision has been the subject of research and development since the 1980s [3] [4] with a legacy of predominantly photographic based systems with widely varying degrees of success and no general solution available on the market. An in-depth review of past photographic based systems is provided by the presenting author [5] [6] but it outside the scope of this paper.

There are a number of sources of error relevant to techniques that measure only what is visible on the surface of a pile and it is necessary to consider these errors in order to ensure a measurement system that can be stable, reliable and repeatable.

Particle delineation error refers to the inaccuracies of determining the correct delineation of all the individual particles in the measured surface (whether by an automatic computer program or manually). Significant error will render the mea- 
sured surface largely meaningless as the particle delineation will bear little resemblance to the reality of what is on the surface of the pile. This error has been quantified by the presenting author at the laboratory scale, but not for this open pit application [7].

Sub-resolution particle error, relates to the inability of an imaging system to see fine particles below the resolution of the sensor. For example, to detect an individual particle that is only represented by a few pixels or less in an image. These sub-resolution particles tend to be grouped into larger regions and mis-sized as large rocks. Detection of these areas of sub-resolution particles (hereafter referred to as areas-offines) is therefore critical to prevent a large error towards larger sizes. The presenting author has published results and algorithms demonstrating this capability for rocks on conveyor [8] and rocks in excavator buckets [9]. Noy [10] [11] published 3D images of rocks and areas-of-fines and stated that the segmentation algorithm could distinguish between rocks and a pile of fines, but no segmentation results or details of this algorithm have been published.

Segregation and grouping error, more generally known as the brazil-nut-effect [12], describes the tendency of the pile to separate into groups of similarly sized particles. It is caused by vibration or motion (for example as rocks are excavated or transported by truck or conveyor) with large particles being moved to the surface. It is advisable to measure at a location before the material has been subjected to excessive vibration and segregation. Avoiding excessive segregation error is one recommendation for measurement on the blasted rock pile as compared to in the excavator bucket.

Overlapped particle error, describes the fact that many particles are overlapped by other particles and only partially visible. A bias to the smaller size classes results if overlapped particles are treated as small non-overlapped and sized using only their visible profile. This error can be overcome in piles of particulate material using classification algorithms based on 3D range data [13] successfully providing $82 \%$ classification accuracy [14].

Capturing error [15] describes the varying probability based on size, that a particle will appear on the surface of the pile. In simple terms, the larger a particle is, the more likely one is to be able to see some part of it on the surface. For example, if a single particle is as large as the height of the pile of material, then it will always be visible, whereas a very fine particle is almost certainly not visible. Thurley [6] has explored capturing error in laboratory rock piles but it remains a source of error in this application.

Profile error, describes the fact that only one side (a profile) of an entirely visible particle can be seen making if difficult to estimate the particles size.

Sample delimitation and extraction error is relevant to all methods sampling from a pile of rocks. Over-coming this error requires the correct delimitation of a random section of the pile, and a correct extraction of particles where only particles whose centre-of-gravity is in-side the delimited region are part of the sample. Refer to Pitard [16] for a more thorough description. In this case the measurement of data sets would have to be performed in a consistent manner, and timing and location of measurement would need to be randomised to eliminate operator bias.

Weight estimation error results from the fundamental difference between non-contact measurement and physical measurement. Size measurement using imaging identifies how many particles are observed, but manual sieving measures the weight of particles in each size class. Therefore it is necessary to have a method of mapping from numbers of particles to weight of particles in order to provide a measurement of size that industry understands and can use. The presented research uses volumetric estimation of each non-overlapped particle and assumes constant density within a sample to estimate a weight of each particle. Weight of fines is estimated based on a bulk volume estimated from the observed areas-of-fines scaled by a constant factor.

To summarise, photographic based 2D imaging systems are subject to significant particle delineation error due to uneven lighting conditions, excessive shadowing, and colour and texture variation in the material. Furthermore, photographic systems have no direct measure of scale, suffer from perspective distortion, lack the capability to distinguish between overlapped and non-overlapped particles, and do not demonstrate the ability to automatically detect areas-of-fines in a realistic way. As a result photographic 2D systems typically require manual editing of the particle delineation to provide a reasonable estimation. As a recent example shows, Petropolous et. al. [17] used a commercial 2D system to evaluate blast fragmentation based on images of the rocks in 234 mining trucks, however all of these 234 images required manual segmentation to specify all the individual rocks. This substantial effort is not unique and highlights the need for a new approach using automated analysis algorithms based on $3 \mathrm{D}$ profile data.

Automated particle size distribution measurement based on $3 \mathrm{D}$ imaging has been the subject of research of the presenting author for over 15 years and has transitioned from laboratory research [18] [7] to industrial prototype [5] [19] and now commercial system [8]. However, the bulk of this work applies to $3 \mathrm{D}$ imaging of material on a conveyor belt, where the environment and lighting is readily constrained and controlled. Application to blasted rock in underground excavators has also been demonstrated [9] but this again allows a high degree of control over lighting and measurement geometry.

This paper is the first time that an automated image analysis strategy has been presented for 3D data of the surface of blasted rock piles in an open-pit in order to measure the particle size distribution of the visible material. Noy [11] recently published particle size distribution results based on stereo photogrammetry images of blasted rock in a pit, but no image analysis strategy is described and no image segmentation results are shown.

Given the difficulties of accurate particle delineation using 2D based photographic methods we restrict the focus to particle size measurement using 3D profile data and note the following additional publications [10, rocks] [11] [20, sugar beets] [21, river rock] [22].

However, only Frydendal [20], and the presenting author [13] have published methods to remove the bias resulting from overlapped particles. Frydendal [20] used graph theory and 
average region height to determine the entirely visible sugar beets but this relied on the regular shape and size of the beets. Only the presenting author has developed an algorithm to distinguish between overlapped and non-overlapped particles using the advantages of 3D range data and in a manner that does not presume constraints on size or shape [13].

The mine operators are interested in understanding the size distribution of the rock pile and particularly the proportion of fines.

Therefore the objectives of the investigation were to;

1) collect data and perform an automatic image segmentation of the rock pile

2) demonstrate the capacity to produce size distribution results that look realistic and are useful for comparison

3) evaluate if the sub-resolution particle detection strategy for areas-of-fines [9] [8] could be successfully applied

4) evaluate what the smallest size of particles is that can practically be identified

The presented paper is the first case study to demonstrate particle size distribution measurement of blasted rock piles using 3D profile data in an open-pit, the presents;

1) the image analysis algorithm, and

2) image analysis results for identifying areas-of-fines.

\section{METHOD}

\section{A. Measurement Hardware}

One of the key criteria for particle size measurement is high data density as it defines the capacity to detect small overlapped particles, the lower limit on particle size that can be reliably detected, and the resolution of size classes detectable.

Therefore data collection was performed using a high resolution laser scanner to collect a dense $3 \mathrm{D}$ point cloud. The device used was a Maptek I-Site 8810 laser scanner. It is a long range time-of-flight laser scanner built into a rugged housing to make it robust for use in a mine. The product specifications states a minimum angular step size of $0.0125^{\circ}$, and a beam divergence of $0.25 \mathrm{mrad}$ (approx. $0.014^{\circ}$ ). The beam divergence is only slightly larger than the angular step size, so the spot size of the laser at any given point will be quite similar to the distance between that point and its neighboring points. A more detailed list of specifications are shown in table I.

Irregularly spaced data 3D points has an impact on both the analysis method, but more importantly the results. Rocks that are further away from the scanner will have fewer measured $3 \mathrm{D}$ points on them, meaning smaller rocks will be less able to be detected the further away they are.

TABLE I. MAPTEK I-Site 8810 LASER SCANNER SPECificATIONS

\begin{tabular}{ll}
\hline Maximum range & Up to $500 \mathrm{~m}$ with reflectivity $>10 \%$ \\
Range accuracy & $10 \mathrm{~mm}$ \\
Range repeatability & $\pm 8 \mathrm{~mm}$ \\
Beam divergence & $0.25 \mathrm{mrad}\left(\right.$ approx. $0.014^{\circ}$ ) \\
Angular step & $0.2^{\circ}$ to $0.0125^{\circ}$ \\
Angular accuracy & $0.01^{\circ}$ \\
Angular scanning range & $80^{\circ}$ vertical, $360^{\circ}$ horizontal \\
\hline
\end{tabular}

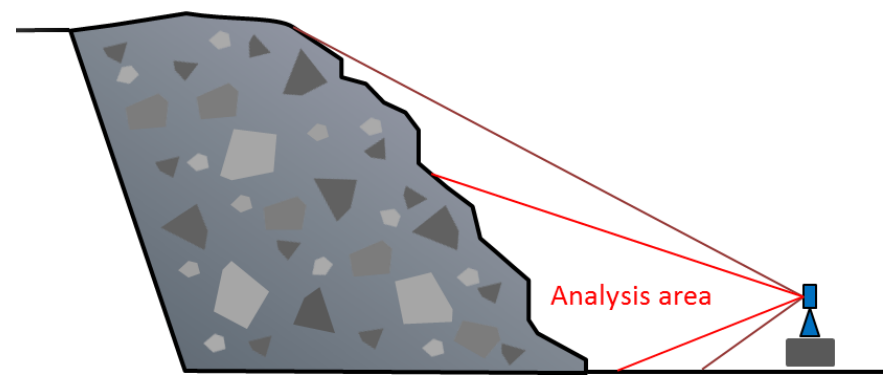

Fig. 1. Measurement geometry

\section{B. Measurement Geometry}

The laser scanner is operated by the mine personnel with the highest angular resolution of $0.0125^{\circ}$. The laser scanner was positioned in front of the rock pile to be measured, and as close to the pile as practical. Based on the collected data sets a distance of 8 to $10 \mathrm{~m}$ appears to have been as close as the mine personnel got to the pile. As the laser scanner is an angular based scanner the euclidean distance between consecutive measured 3D points increases as the angle between the laser and the vector between the scanner and the closest point on the rock pile increases. To extract the data with the highest spatial resolution the data was cropped to extract the central part where the 3D data points are closer together. The data was cropped between $\pm 12^{\circ}$ in the horizontal plane, and approximately $-5^{\circ}$ to $+12^{\circ}$ in the vertical plane. Furthermore, a vertical section $500 \mathrm{~mm}$ high has been cropped off the bottom of the data to remove the floor of the pit. Figure 1 shows the setup of the scanner in the open-pit. There is no special reason for choosing precisely $\pm 12^{\circ}$, but it was chosen as a balance between a high spatial resolution and having a large enough view of the rocks on the pile.

Consider a sample cropped measured data set with $2,434,727$ points (shown in figure 2) which we will denote, set $\mathrm{A}$, and that it exists in a right handed $3 \mathrm{D}$ coordinate system with the positive $\mathrm{z}$ axis being a horizontal line from the rock pile to the laser scanner. Consider the $x, y$ plane equivalent to an image plane with non-regular spacing of points. The data set has fixed angular resolution but an increasing spatial resolution $(\Delta x$ and $\Delta y)$ in the image plane between neighboring 3D points. Set A has an extent of $[-4.657 \mathrm{~m}, 4.752 \mathrm{~m}]$ in the $\mathrm{x}$ axis, [-1.689m, $5.304 \mathrm{~m}]$ in the $y$ axis, and [-24.923m, $-9.311 \mathrm{~m}]$ in the $\mathrm{z}$ axis, where the laser scanner is positioned at the origin. Using trigonometry the distance measures between neighboring points can be considered in the simple case. At a distance of $10 \mathrm{~m}$ from a vertical wall, with a horizontal angle of $0^{\circ}$, and vertical angles of $0^{\circ}$ and $0.0125^{\circ}$ the $\Delta y$ distance is $2.2 \mathrm{~mm}$. If the vertical angles are increased to $11.9875^{\circ}$ and $12^{\circ}$ then the $\Delta y$ distance is $2.3 \mathrm{~mm}$. But this is the best case of a perpendicular surface to the scanner and the worst case is less trivial as the scanner is measuring values on the rock pile which is inclined away in the $y$ axis.

Given the known extents of set A, we convert figure 1 into a simple geometric representation of the $y-z$ plane as shown in figure 3 . This figure depicts the laser scanner at the origin $O$, the rock pile approximated by the triangle $\triangle Q_{1} P_{1} B$, and the dashed line $\overline{O Q_{2}}$ from the laser scanner intersecting the 


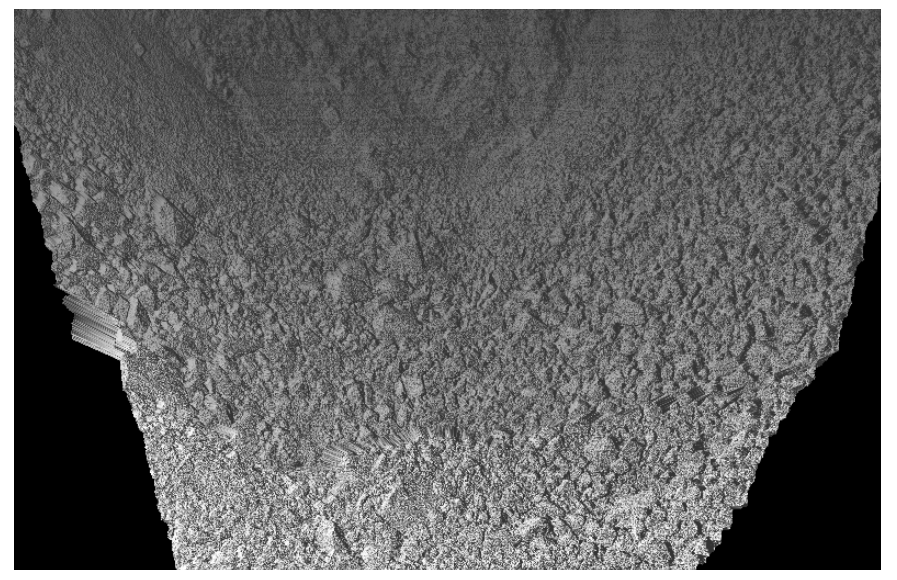

Fig. 2. Set A - Cropped 3D surface data of a blasted rock pile (9.4m wide)

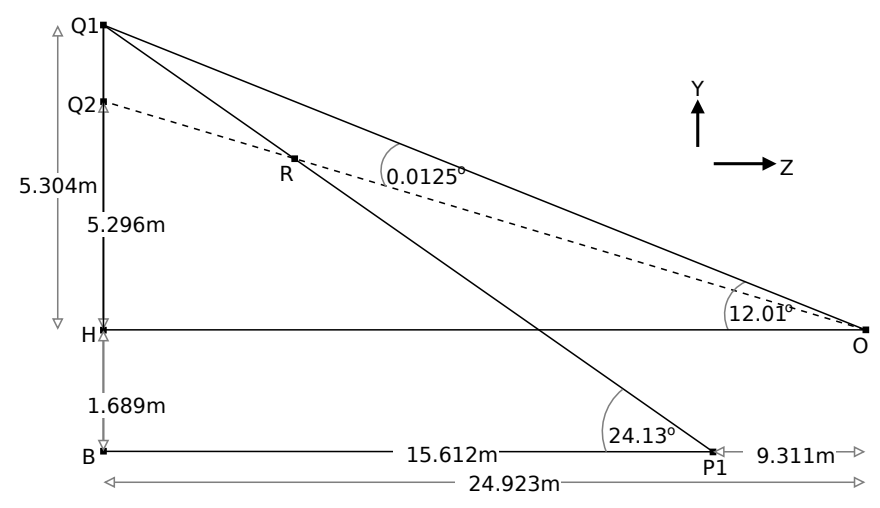

Fig. 3. Measurement geometry for an example measured data set (set A)

surface of the rock pile at $R$. Using simple trigonometry the length of the line $\overline{H Q_{2}}$, and the angles $\angle Q_{1} O H$, and $\angle Q_{1} P_{1} B$ have been pre-calculated. Although the angle $\angle Q_{1} O H$ is not exactly $12^{\circ}$ as expected it is within the angular accuracy given in table I. What is of interest is the maximum $\Delta y$ which will be found between points $R$ and $Q 1$. Using the parametric equations of a line the intersection of the lines $\overline{O Q_{2}}$ and $\overline{P_{1} Q_{1}}$ can be calculated. The result is that $R$ is positioned at $(\mathrm{z}, \mathrm{y})=(-$ $24.889 \mathrm{~m}, 5.289 \mathrm{~m})$, and the $\Delta y$ between $R$ and $Q_{1}$ is therefore $15 \mathrm{~mm}$ and the length of the line $\overline{R Q_{1}}$ is $37 \mathrm{~mm}$.

From experience of analysing 3D profile data of piled particles [8] [9] [19], the expectation is that particles in a pile can be reliably detected at a size of approximately 10 times the spacing between points in the image plane. Therefore, for set A with $3 \mathrm{D}$ data points with a $\Delta y$ ranging between $2.2 \mathrm{~mm}$ and $15 \mathrm{~mm}$, the expectation is to detect particles larger than $22 \mathrm{~m}$ at the area of highest point density, and larger than $150 \mathrm{~mm}$ in the areas of lowest point density. Unfortunately this upper limit is higher than would be preferred. The only ways to improve this with the current scanner is to change the measurement geometry by being closer to the pile.

The beam divergence of the laser is only slightly larger than the angular resolution, so we can expect that the beam spot size is similar to the values calculated here, with a beam size of the surface of the pile slightly larger than $37 \mathrm{~mm}$ in the worst case. The implication being that the $3 \mathrm{D}$ point calculated in this case is some weighted averaged value of the rocks in a circular area slightly larger than $37 \mathrm{~mm}$ diameter. This will add a smoothing to the data, blurring the edges and likely making particle segmentation more difficult in these areas.

\section{Pre-processing}

The data is preprocessed to convert it onto a regular grid. This begins by performing an orthogonalisation (or perspective normalisation) in both the $\mathrm{y}$ and $\mathrm{x}$ axes using the following equations, where the $\mathrm{k}$ values rescale the data back to the same $\mathrm{x}, \mathrm{y}$ extent as from before normalisation;

$$
\begin{aligned}
x^{\prime} & =-k_{x} * x / z \\
y^{\prime} & =-k_{y} * y / z
\end{aligned}
$$

Before orthogonalisation the average spacing between points for set A $4.3 \mathrm{~mm}$ in $\Delta x$ and $4.7 \mathrm{~mm}$ in $\Delta y$. After orthogonalisation the average spacing between neighboring points is $5.4 \mathrm{~mm}$ in $\Delta x$ and $4.3 \mathrm{~mm} \Delta y$. The larger of these values is used to select a spacing value for the regular grid.

The data is resampled onto a regular grid using a grid spacing based on the larger of the average $\Delta x$ and $\Delta y$ spacing values after orthogonalisation. The image segmentation and morphological image processing algorithms used were implemented to handle incomplete data sets [7] so it is not necessary to resample to a complete grid, or interpolate the grid to fill in missing values. 3D points are binned into the regular grid and where multiple values occur in a single bin these are averaged. A resampling method using no additional smoothing was chosen to preserve as much as possible the sharp edges present in the rock pile data.

In the case of set $\mathrm{A}$, the regular grid is $5.4 \mathrm{~mm}$ between points, has 1199 rows, 1737 cols, and although this could contain a maximum of $2,082,663$ points in the grid, set $A$ contains only $1,870,594$ points.

The implications of orthogonalising the data are that neighborhood operations are performed on a number of neighboring points, which means that for a neighborhood closer to the scanner, the neighborhood will be smaller in size $(\mathrm{mm})$ than for a neighborhood further away. The advantage of this is that the data density can be exploited to its full resolution allowing for the detection of small particles when there is sufficiently high resolution to do so.

\section{Segmentation}

The segmentation approach is based on watershed segmentation and morphological operators. These operations can be found in most textbooks on image processing, for example, the following accessible text on morphological image processing by Dougherty and Lotufo [23].

Filtering is performed to remove the erroneous rays of data points that can occur in the laser data. These rays of $3 \mathrm{D}$ data points appear in vertical columns of data and arc up from the rock pile surface and remain above the pile surface. These are removed using a minimum filter, a morphological opening by reconstruction with a spherical structuring element of diameter 9 times the grid spacing, and a reconstruction using a circular structuring element with diameter 3 times the grid spacing. Recall that in the case of set A, the grid spacing is $5.4 \mathrm{~mm}$. 


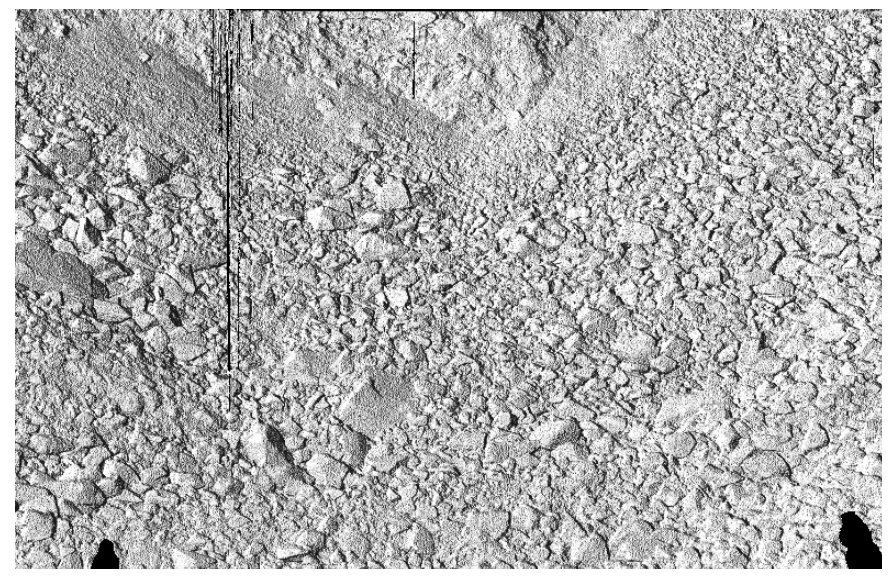

Fig. 4. Set A - Orthogonalised data (9.4m wide)

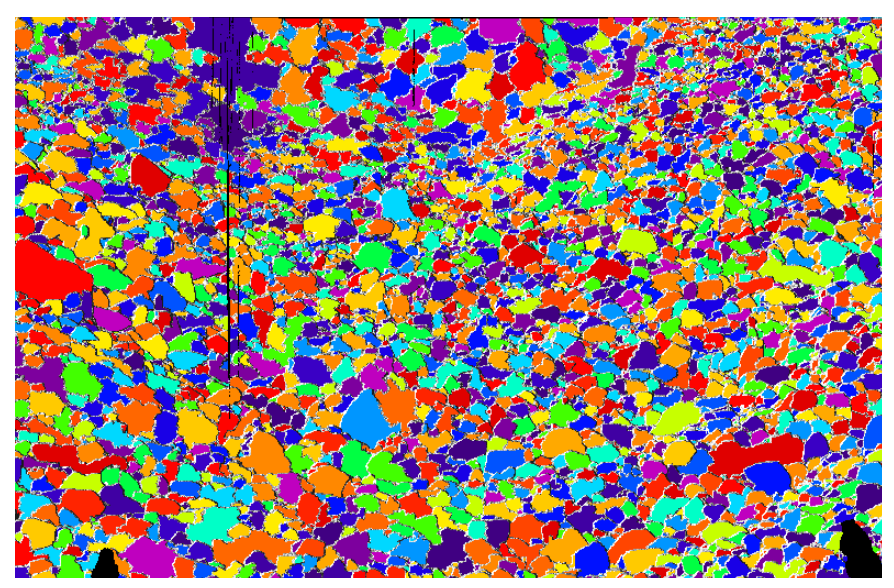

Fig. 5. Set A - Segmentation of the Orthogonalised data

Edge detection is performed using a morphological gradient with a spherical structuring element with diameter of 3 times the grid spacing. A threshold of $60 \mathrm{~mm}$ is used to classify edges.

Seed formation for the watershed segmentation is performed using a three step process [5, 3.1.2 Seed formation] based on distance transform, local maxima, and seed merging. Part one is a geodesic distance transform [23] applied to approximate the distance between non-edge points and the nearest edge point. This is a fast morphological reconstruction based approximation to the distance transform [24]. Part two detects the local maxima in the distance transform, and part three uses these local maxima and the distance transform values to create seed regions. Seed regions are formed by creating and merging circular disks based on the position and distance of each local maxima in the distance transform [7, 7.7 Calculating the seed regions].

Watershed segmentation based on the seed regions is applied to the morphological gradient (calculated earlier) of the rock pile data after which a filter is applied to remove small regions with fewer than 25 data points.

Figure 5 shows the resultant segmentation for figure 4 and figures 6 and 7 show two magnified areas of the segmentation.

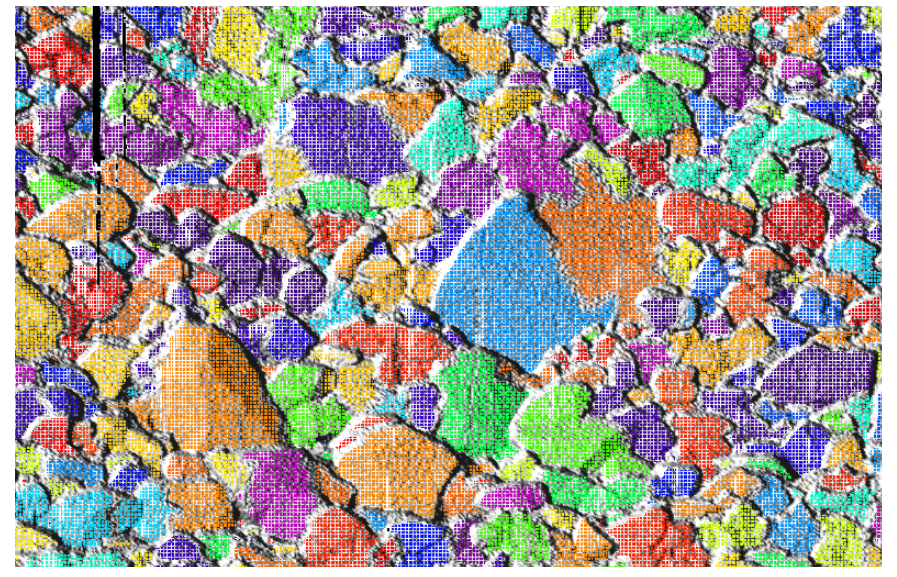

Fig. 6. Set A - Magnified area of the segmentation

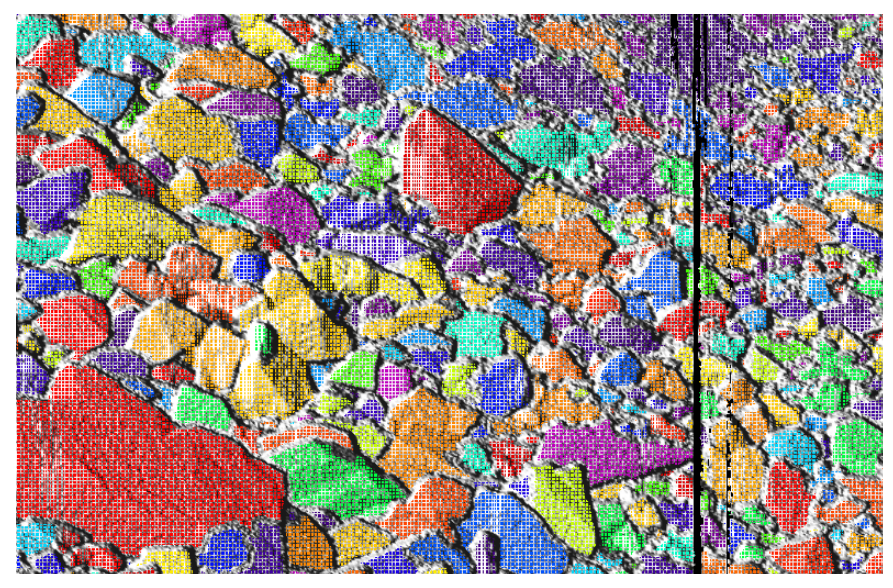

Fig. 7. Set A - Magnified area of the segmentation

\section{E. Particle Classification}

Particle classification assigns each region in the segmentation to be either a non-overlapped particle, an overlapped particle, or an area-of-fines.

An area-of-fines on the rock pile is comprised of many small particles too small to individually detect in the segmentation. It is typically smoothly varying in height and free from large height discontinuities due to how particles lie at rest in a pile. Larger rocks exhibit large height discontinuities around their edges.

The classification features are based on algorithms developed by the presenting author [13] [9]. These algorithms examine each region in the segmentation and perform a neighborhood based analysis of the height information around the perimeter of each particle, as summarised below.

A series of prominent points spaced around the perimeter of each region are calculated.

A neighborhood analysis is performed around each of these perimeter points calculating the height of the "current" region in this perimeter neighborhood and the height of points not part of this region.

A visibility ratio feature [13] is calculated based on how often the "current" region in perimeter neighborhood is above 
or below neighboring regions. The ratio is between 0 for all perimeter neighborhoods below, and 1 for all above.

An average absolute height difference (AAHD) feature [9] is calculated to show the height difference in each perimeter region between the "current" region and neighboring regions. For areas-of-fines where the 3D surface is relatively smooth, this feature will be closer to 0 , and for edges of rocks with large height discontinuities this feature will be larger.

The segmentation strategy described above was not developed on set A. It was developed using 4 other data sets. One of these sets (denoted set D) is shown in figure 8 was used to create a classification strategy for areas-of-fines. Manual editing was performed to classify parts of the data set into areas-of-fines as shown in figure 8. This was not intended to identify all areas-of-fines in this set, simply a large portion of fines in different areas to make a useful classifier. Set D was segmented, the visibility ratio and AAHD feature were calculated, and the AAHD feature was plotted for all regions based on their manual classification into area-of-fines as shown in figure 9. The red line in figure 9 shows a decision boundary at an AAHD value of 74 dividing the two populations at an equivalent percentile, excluding slightly more then the top quartile of the areas-of-fines, and including slightly more than the bottom quartile of larger rocks into the areas-of-fines.

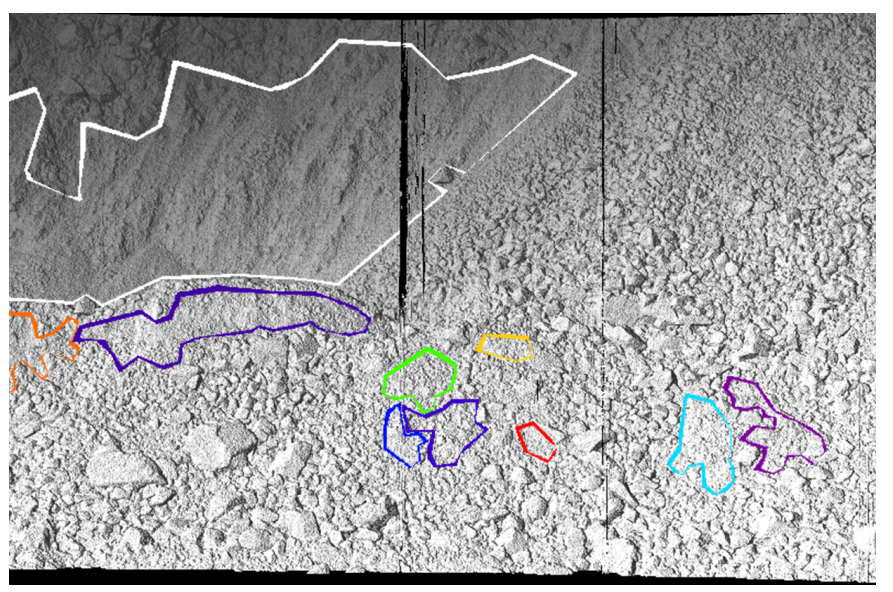

Fig. 8. Set D - Orthogonalised data ( $15.4 \mathrm{~m}$ wide) with selected areas-of-fines

\section{AAHD feature for areas-of-fines and other-rocks}

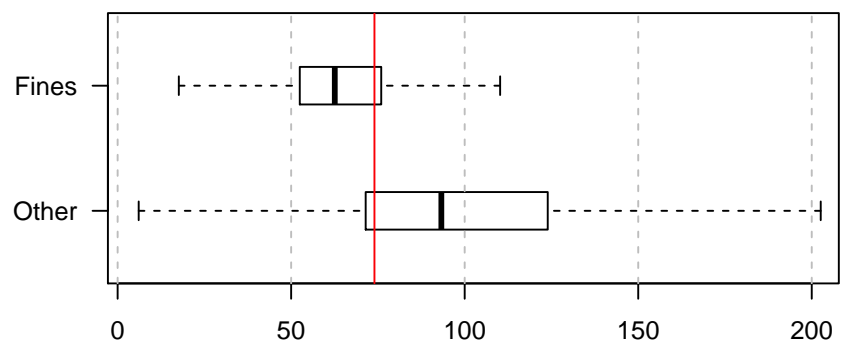

Fig. 9. AAHD height feature for manually classified areas-of-fines. Red line decision boundary at $\mathrm{AAHD}=74$

The remaining rocks are classified into either overlapped or non-overlapped rocks. Algorithms to achieve this classification to mitigate overlapped particle error, were developed on laboratory rock piles [13] and applied to industrial measurement on conveyor belt [5] [8]. In each of these cases the rock pile is significantly constrained geometrically, and the measurement of the pile is essentially perpendicular to the pile surface. The measurements on the rock piles in the open-pit are at an acute angle to the surface of the pile, and this somewhat disconnects the observation of a rock being above or below in the 3D data (from the point of view of the laser scanner) from whether the rock is siting on the surface or not. An elevated measurement position would likely be better for overlapped/non-overlapped classification. However, the visibility ratio feature has been demonstrated as a successful classifier for this purpose [14] and is applied here. An ad-hoc selection of decision boundary of 0.45 for the visibility ratio has been chosen simply based on visual assessment of the four development data sets, one of which is shown in figure 8 .

\section{F. Sizing}

Before sizing an inverse orthogonalisation is applied to the $3 \mathrm{D}$ data to map it back into the actual dimensions of the rock mass.

A volumetric based sizing approach is applied, estimating the volume of individual rocks, and areas-of-fines, and assuming constant density to calculate an approximation of a cumulative percent passing by mass graph required by the mine personnel.

Each region identified as fines is converted into a volume based on the 3D surface profile and a fixed depth scaling factor of $250 \mathrm{~mm}$. The fines volume is allocated to a preset minimum value of 5 times the grid spacing, or approximately $25-30 \mathrm{~mm}$.

Each region identified as overlapped is ignored. These regions are analogous to ice-bergs and are a more difficult prospect for sizing. Identifying and ignoring these particles prevents mis- sizing them are smaller particles.

Each region identified as non-overlapped is processed as follows;

- The best-fit-rectangle in the horizontal plane is calculated for that region. From the length and width of this rectangle a major axis and a minor axis for the particle is defined, denoted $\alpha, \beta$ respectively.

- The height is approximated as equal to the width $\beta$. Whereas in the conveyor belt application, the partial height profile of the rock is useful to help estimate the third dimension of the rock, in this case the height information is very susceptible to small segmentation errors introducing large height variations and error.

- The volume of the particle is approximated using an ellipsoid with axes major, minor, minor. Therefore the volume $V=(4 / 3) \pi \alpha \beta^{2}$

- The sieve-size of a particle is approximated as the minor axis $\beta$ as this is the side length of the square mesh the ellipsoid would fit through.

- The cumulative sieve-size-distribution is calculated by ordering all of the particles by their sieve-size (starting with the fines volume) and calculating the cumulative sum of all of their volumes. 


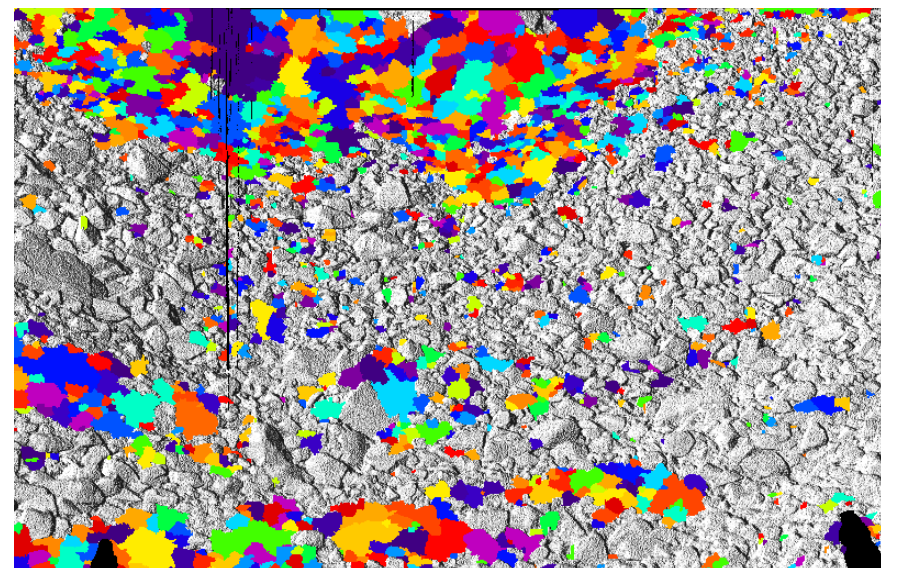

Fig. 10. Set A - Regions classified as areas-of-fines

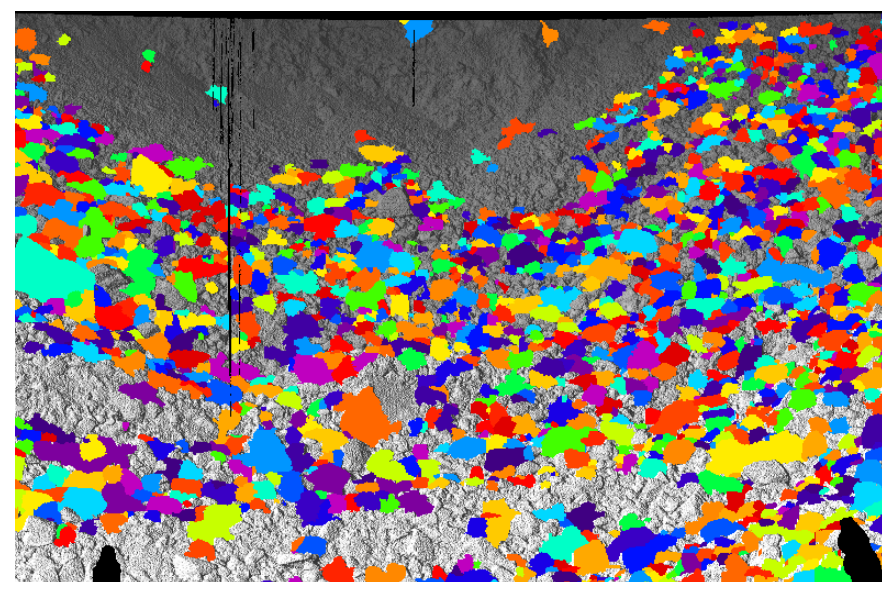

Fig. 11. Set A - Regions classified as non-overlapped rocks

\section{RESULTS}

Using the classification strategies for areas-of-fines and overlapped/non-overlapped rocks, a number of data sets, denoted A, B, C were classified. Sets B and C were chosen to provide examples with proportionately more large rocks. From a visual analysis of the 3D data it is clear that set $\mathrm{B}$ contains a high proportion of larger rocks, set $\mathrm{C}$ also contains large rocks but also many smaller rocks, and set $\mathrm{A}$ contains a very high proportion of material that we expect to classify as fine particles. Figure 10 shows the regions classified as areas-of-fines overlayed on the set A from figure 2. This result is especially encouraging, as the top section of the rock pile was identified by the mine personnel as a large section of highly compacted powder like fines, with additional fines concentrated in the front and left side of the pile. Figure 11 shows the regions identified as non-overlapped rocks.

Figure 14 depicts a cumulative size distribution graph for set $A$ (figure 2, set B (figure 12), and set C (figure 13). It is clear from looking at these figures that set $\mathrm{A}$ has far more small rocks and areas-of-fines than the other sets and that is represented in the size distribution graph. Set B contains many more larger rocks and this is again represented in the graph. From this small sample of results it appears that the segmentation and sizing approach can produce sizing results that show a realistic comparative result.

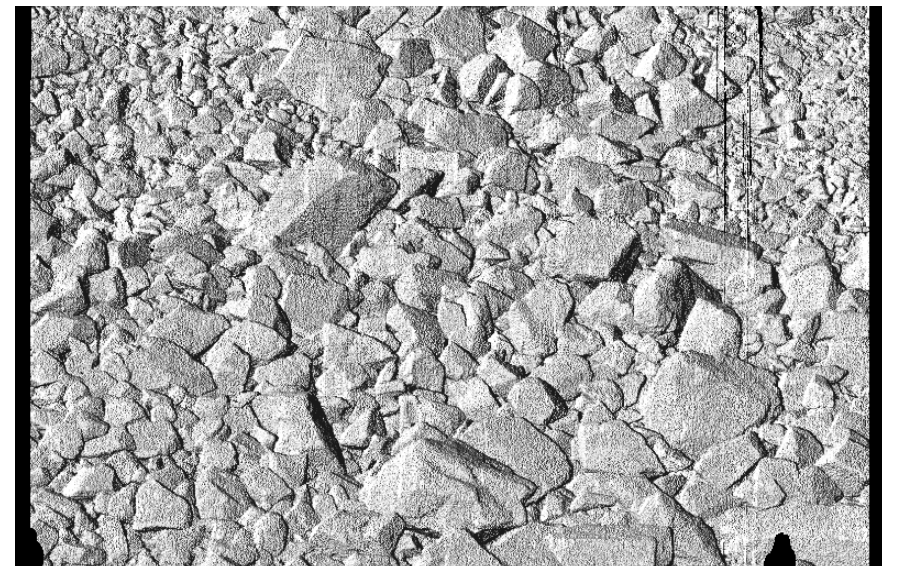

Fig. 12. Set B - Orthogonalised data ( $7.3 \mathrm{~m}$ wide $)$

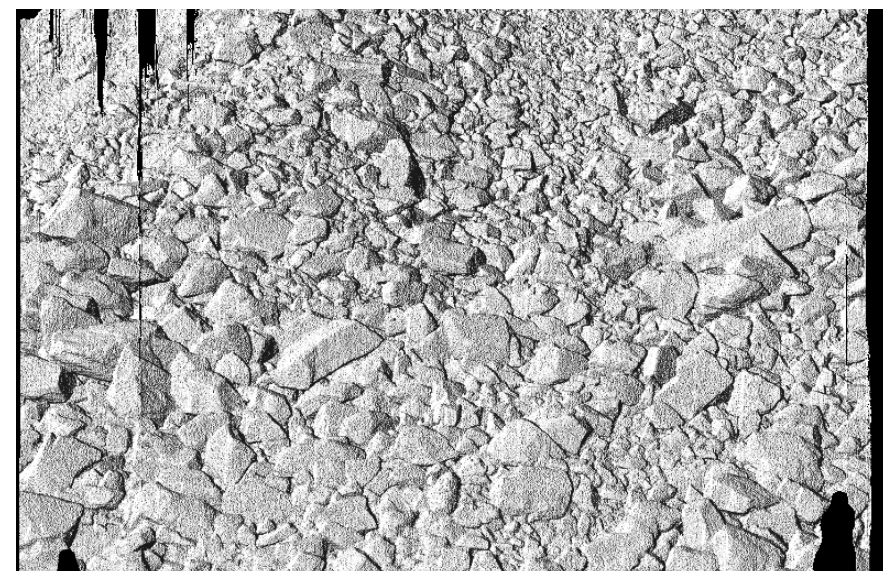

Fig. 13. Set C - Orthogonalised data ( $8.8 \mathrm{~m}$ wide)

\section{DISCUSSION \& CONCLUSION}

The stated objectives were four-fold and have substantially been achieved by the presented work. Using the Maptek laser scanner it is practical to collect data of the open-pit rock piles and perform an automatic analysis to determine a size distribution.

Furthermore, the calculated results provide a useful comparative result where variation in material size is clear in the comparison of sets A, B, and C. Due to various sources of error, particularly capturing error, segregation error, profile error, and others, there is not an expectation that this technique, or any other technique based on measurement of the surface of a rock pile, will produce a measurement that is equivalent to manual sieving/screening.

The work has demonstrated that the classification strategy can detect areas-of-fines / sub-resolution particles in the data. Testing on additional data sets is required and there is clearly significant scope for using more advanced multi-feature classification, and robust selection of parameters in general.

From observation of the cumulative size distribution graph, and the size results, it is clear that a relatively low estimated mass of particles larger than the fines size but smaller than $80-100 \mathrm{~mm}$ are being detected. The flattening of the graph curves in the tail is not an expected phenomena from the 


\section{Open Pit Size Distributions}

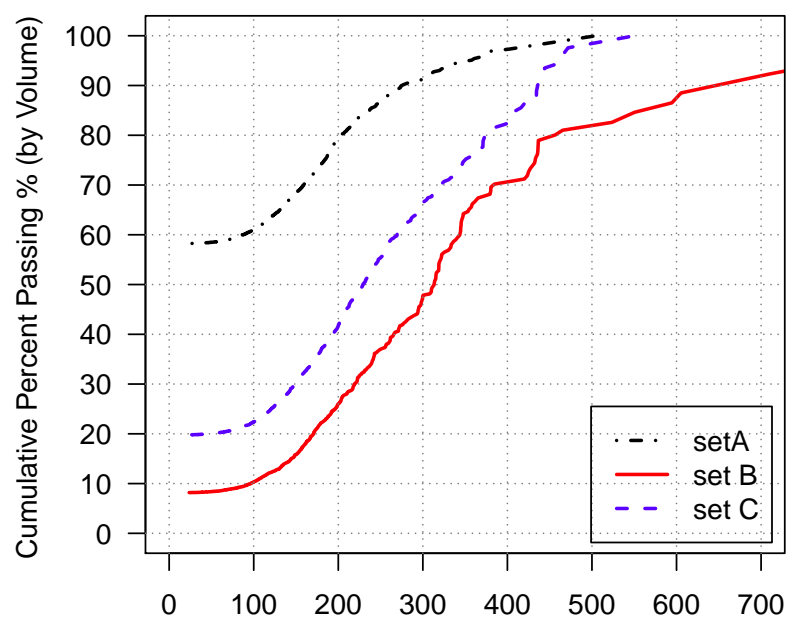

Fig. 14. Cumulative size distribution curve for set A, B, and C

mining personnel point-of-view and indicates that either there are insufficient small particles visible on the surface of the piles measured, or that the resolution was insufficient to detect enough of these smaller particles.

\section{ACKNOWLEDGMENT}

The author would like to thank Italo Onederra and his partners for undertaking and coordinating the project. Furthermore, the author wishes to thank the INTERREG IVA A Nord programme of the European Union for partially supporting the work, and Luleå University of Technology for their ongoing support.

\section{REFERENCES}

[1] A. MacKenzie, "Cost of explosives - Do you evaluate it properly?" Mining Congress Journal, pp. 32-41, May 1966.

[2] J. Grant, T. Little, and D. Bettess, "Blast driven mine optimisation," in Explosives in Mining Conference. Brisbane, Australia: AusIMM, September 1995, pp. 3-10.

[3] O. Carlsson and L. Nyberg, "A method for estimation of fragmentation size distribution with automatic image processing," in Proceedings of the First International Symposium on Rock Fragmentation by Blasting - FRAGBLAST, Luleå, Sweden, August 1983, pp. 333-345.

[4] A. Ord, "Real-time image analysis of size and shape distributions of rock fragments," in Explosives in Mining Workshop. Melbourne: The AusIMM, November 1988, pp. 115-119.

[5] M. J. Thurley, "Automated online measurement of limestone particle size distibutions using 3D range data," Journal of Process Control, vol. 21, no. 2, pp. 254-262, 2011.

[6] M. J. Thurley, "Three dimensional data analysis for the separation and sizing of rock piles in mining," Ph.D. dissertation, Monash University, December 2002. [Online]. Available: http://image3d6.eng.monash.edu. au/thesis.html

[7] M. J. Thurley and K. C. Ng, "Identifying, visualizing, and comparing regions in irregularly spaced 3D surface data," Computer Vision and Image Understanding, vol. 98, no. 2, pp. 239-270, February 2005.

[8] M. J. Thurley, "Automated, on-line, calibration-free, particle size measurement using 3D profile data," in Measurement and Analysis of Blast Fragmentation: Workshop at FRAGBLAST 10 - The 10th International Symposium on Rock Fragmentation by Blasting. Dehli, India: CRC Press/Balkema, November 2012, pp. 23-32, ISBN 978-041562140-3.
[9] M. J. Thurley, "Fragmentation size measurement using 3D surface imaging (in LHD buckets)," in Proceedings of the Ninth International Symposium on Rock Fragmentation by Blasting - FRAGBLAST 9, Granada, Spain, September 2009, pp. 133-140.

[10] M. J. Noy, "The latest in on-line fragmentation measurement - stereo imaging over a conveyor," in Proceedings of the Eighth International Symposium on Rock Fragmentation by Blasting - FRAGBLAST 8, May 2006, pp. 61-66.

[11] M. J. Noy, "Automated rock fragmentation measurement with close range digital photogrammetry," in Measurement and Analysis of Blast Fragmentation : Workshop at FRAGBLAST 10 - The 10th International Symposium on Rock Fragmentation by Blasting. Dehli, India: CRC Press/Balkema, November 2012, pp. 12-21, ISBN 978-041562140-3.

[12] A. Rosato, K. Strandburg, F. Prinz, and R. Swendsen, "Why the brazil nuts are on top: Size segregation of particulate matter by shaking," Physical Review Letters, vol. 58, no. 10, pp. 1038-1040, 1987.

[13] M. J. Thurley and K. C. Ng, "Identification and sizing of the entirely visible rocks from segmented 3D surface data of laboratory rock piles," Computer Vision and Image Understanding, vol. 111, no. 2, pp. 170178, August 2008.

[14] T. Andersson and M. J. Thurley, "Visibility classification of rocks piles," in Proceedings of the 2008 Conference of the Australian Pattern Recognition Society on Digital Image Computing Techniques and Applications (DICTA 2008). Australian Pattern Recognition Society, December 2008, pp. 207-213.

[15] R. Chavez, N. Cheimanoff, and J. Schleifer, "Sampling problems during grain size distribution measurements," in Proceedings of the Fifth International Symposium on Rock Fragmentation by Blasting FRAGBLAST 5, Montreal, Quebec, Canada, August 1996, pp. 245-252.

[16] E. Pitard, Pierre Gy's sampling theory and sampling practice: heterogeneity, sampling correctness and statistical process control. CRC Press, 1993.

[17] N. Petropoulos, D. Johansson, U. Nyberg, E. Novikov, and A. Beyglou, "Improved blasting results through precise initiation Results from field trials at the aitik open pit mine," Swedish Blasting Research Centre at Luleå University of Technology, Tech. Rep., March 2013, ISSN 1653-5006. [Online]. Available: http://pure.ltu.se/portal/files/42740636/Swebrec_2013_1_web.pdf

[18] M. J. Thurley, K. C. Ng, and J. Minack, "Mathematical morphology implemented for discrete, irregular 3D surface data," in DICTA 99, Digital Image Computing: Techniques and Applications Conference Proceedings, ser. Fifth Biennial Conference. Curtin University, Perth, Western Australia: Australian Pattern Recognition Society, December 1999, pp. 16-20.

[19] M. J. Thurley and T. Andersson, "An industrial 3D vision system for size measurement of iron ore green pellets using morphological image segmentation," Minerals Engineering, vol. 21, no. 5, pp. 405-415, 2007.

[20] I. Frydendal and R. Jones, "Segmentation of sugar beets using image and graph processing," in ICPR 98 Proceedings - $14^{\text {th }}$ International Conference on Pattern Recognition, vol. II, Brisbane, Australia, August 1998, pp. 16-20.

[21] H. Kim, C. Haas, A. Rauch, and C. Browne, "3D image segmentation of aggregates from laser profiling," Computer Aided Civil and Infrastructure Engineering, pp. 254-263, 2003.

[22] J. Lee, M. Smith, L. Smith, and P. Midha, "A mathematical morphology approach to image based 3D particle shape analysis," in Machine Vision and Applications, vol. 16(5). Springer-Verlag, 2005, pp. 282-288.

[23] E. R. Dougherty and R. A. Lotufo, Hands-On Morphological Image Processing. SPIE - The International Society for Optical Engineering, 2003, vol. TT59.

[24] G. Borgefors, "Distance transforms in digital images," Computer Vision, Graphics, and Image Processing, vol. 34, pp. 344-371, 1986. 